\title{
Guinea pig transglutaminase immunolinked assay does not predict coeliac disease in patients with chronic liver disease
}

\author{
A Carroccio, L Giannitrapani, M Soresi, T Not, G Iacono, C Di Rosa, E Panfili, \\ A Notarbartolo, G Montalto
}

\begin{abstract}
Background-It has been suggested that serological screening for coeliac disease (CD) should be performed in patients with chronic unexplained hypertransaminasaemia.

Aims-To evaluate the specificity for CD diagnosis of serum IgA antitissue transglutaminase (tTG) determination in consecutive patients with chronic hypertransaminasaemia using the most widely utilised ELISA based on TTG from guinea pig as the antigen.
\end{abstract}

Patients and methods-We studied 98 patients with chronic hypertransaminasaemia, evaluated for the first time in a hepatology clinic. Serum anti-tTG and antiendomysial (EmA) assays were performed. Patients positive for $\mathrm{EmA}$ and/or anti-tTG were proposed for intestinal biopsy. Finally, all sera were reassayed for anti-tTG using an ELISA based on human recombinant $\mathrm{tTG}$ as the antigen.

Results-A total of 94/98 hypertransaminasaemic patients were positive for hepatitis virus markers, with $82 / 98 \quad(83 \%)$ positive for anti-hepatitis $C$ virus. Liver histology showed that most patients had mild or moderate chronic hepatitis while severe fibrosis or overt liver cirrhosis was found in 20/98. CD screening showed that $15 / 98$ (16\%) hypertransaminasaemic subjects had anti-tTG values in the same range as CD patients; however, IgA EmA were positive in only $2 / 98(2 \%)$. Distal duodenal biopsy, performed in nine patients, showed subtotal villous atrophy in the two EmA+/anti-tTG+ patients but was normal in $7 / 7 \mathrm{EmA}$-lanti-tTG+ subjects. The presence of anti-tTG+ values in EmA- patients was unrelated to particular gastrointestinal symptoms, other associated diseases, severity of liver histology, or distribution of viral hepatitis markers. There was a significantly higher frequency of positive serum autoantibodies (antinuclear, antimitochondrial, antismooth muscle, and anti-liver-kidney microsomal antibodies) in anti-tTG+/EmA- patients than in the other subjects (9/13 $v$ 10/83; $\mathbf{p}<0.003)$. Also, a correlation was found between serum gamma globulin and antitTG values $(p<0.01)$. When sera were tested with the ELISA based on human tTG as the antigen, no false positive results were observed: only the two $\mathrm{EmA}+$ patients with atrophy of the intestinal mucosa were positive for anti-tTG while all others were negative, including those false positive in the ELISA based on guinea pig tTG as the antigen.

Conclusions-In patients with elevated transaminases and chronic liver disease there was a high frequency of false positive anti-tTG results using the ELISA based on tTG from guinea pig as the antigen. Indeed, the presence of anti-tTG did not correlate with the presence of EmA or CD. These false positives depend on the presence of hepatic proteins in the commercial tTG obtained from guinea pig liver and disappear when human tTG is used as the antigen in the ELISA system. We suggest that the commonly used tTG ELISA based on guinea pig antigen should not be used as a screening tool for CD in patients with chronic liver disease.

(Gut 2001;49:506-511)

Keywords: liver disease; coeliac disease; antitissue transglutaminase antibodies; antiendomysial antibodies; autoimmunity; intestinal histology

Hepatic damage is a frequent finding in patients with coeliac disease (CD) on a gluten containing diet; in fact, hypertransaminasaemia has been reported in $10-54 \%$ of patients at CD diagnosis. ${ }^{1-4}$ As a consequence, it has been suggested that serological screening for CD should be performed in patients with chronic unexplained hypertransaminasaemia. ${ }^{5}$ In this respect, a previous study revealed that the serum antiendomysial antibody (EmA) assay is the optimum test for predicting CD in patients with chronic liver disease ${ }^{6}$ but this indirect immunofluorescence test is not easy to apply in large scale screening. However, tissue transglutaminase (tTG) has recently been identified as the main (or sole) autoantigen recognised by EmA in CD patients ${ }^{7}$ and this has permitted the use of an ELISA test, based on commercial guinea pig tissue transglutaminase, to detect the presence of anti-tTG autoantibodies in

Abbreviations used in this paper: $\mathrm{CD}$, coeliac disease; EmA, antiendomysial antibody; tTG, tissue transglutaminase; AST, aspartate aminotransferase; ALT, alanine aminotransferase; HCV, hepatitis $\mathrm{C}$ virus; ANA, antinuclear antibodies; AMA, antimitochondrial antibodies; ASMA, antismooth muscle antibodies; anti-LKM, anti-liver-kidney microsomal antibodies; h-tTG, human recombinant tissue transglutaminase; PBS, phosphate buffered saline; IEL, intraepithelial lymphocyte. 
serum, specific for the diagnosis of $\mathrm{CD} \cdot .^{7-11}$ Previous studies on the clinical utility of anti-tTG determination have reported no or very rare false positive results for $C D$ diagnosis, ${ }^{7-11}$ and when positive results have been found in patients with normal intestinal histology, the hypothesis of latent CD has been advanced. ${ }^{8}{ }^{9}$ However, as in the case of patients with chronic liver disease (primary biliary cirrhosis), the occurrence of false positive antitTG antibody results has been found. ${ }^{12}$

In the present study, we evaluated serum anti-tTG and EmA in consecutive patients with chronic hypertransaminasaemia due to various causes.

\section{Patients and methods}

The study included 98 consecutive subjects (66 males; age range 18-64 years, median 36) with chronic hypertransaminasaemia (serum aspartate aminotransferase (AST) and alanine aminotransferase (ALT) levels $>40$ IU/1 for more than two months) who attended the outpatient clinic for liver disease at the Internal Medicine Division of the University Hospital of Palermo for the first time between September 1998 and May 1999. Patients previously hospitalised in our division or examined in our outpatient clinics for liver or gastrointestinal diseases were excluded; we also excluded subjects with known CD and those who in the past had undergone complete serological evaluation for CD diagnosis.

In all patients, alcohol intake, use of drugs, and exposure to potential hepatic toxins were investigated. Laboratory investigations included routine liver and kidney function tests. Immunoglobulin levels were evaluated to exclude IgA deficiency. Furthermore, all subjects underwent serological screening for viral hepatitis B and C (HCV); anti-HCV immunoreactivity was confirmed by a third generation immunoblot assay (RIBA 3; Chiron Corporation, Emeryville, California, USA, and Ortho Diagnostic Systems). Sera were also tested for hepatitis B surface antigen using a commercial ELISA (Abbott Diagnostic, North Chicago, Illinois, USA). In all subjects the presence of antinuclear (ANA), antimitochondrial (AMA), antismooth muscle (ASMA), and anti-liverkidney microsomal (anti-LKM) antibodies was also evaluated by indirect immunofluorescence using commercial kits.

All patients underwent percutaneous liver biopsy with a Menghini needle in accordance with the procedures and precautions previously described. ${ }^{13}$ Liver histological evaluation was performed according to Desmet and colleagues. ${ }^{14}$

Serological screening for CD was performed in all patients by serum EmA and anti-tTG assays based on tTG from guinea pig as the antigen, in accordance with the methods described below. Subjects with positive serum $\mathrm{EmA}$ and/or anti-tTG were asked to undergo intestinal biopsy and commence a gluten free diet to confirm the suspected CD diagnosis.

In the second part of the study we reevaluated all sera for anti-tTG antibodies with a new ELISA based on human recombinant
tTG (h-tTG) as antigen, according to the methods described below.

Control sera for evaluation of anti-tTG antibodies were obtained from two different groups: the first group included $20 \mathrm{EmA}$ positive coeliac patients on a gluten containing diet with total or subtotal intestinal villous atrophy, diagnosed according to the criteria of the European Society of Pediatric Gastroenterology and Nutrition ${ }^{15}$; the second group of control sera was obtained from $35 \mathrm{EmA}$ negative healthy subjects who were members of the medical and laboratory personnel of our clinic. Subjects in these control groups were sex and age matched with the hypertransaminasaemic patients.

All subjects gave informed consent and the protocol was approved by the ethics committee of our hospital.

SERUM ANTIENDOMYSIAL ANTIBODY

DETERMINATION

As previously described, ${ }^{16}$ IgA class EmA values were determined using a commercially available indirect immunofluorescence technique (Anti-endomisio; Eurospital Pharma, Trieste, Italy).

SERUM ANTI-tTG ELISA DETERMINATION USING tTG FROM GUINEA PIG AS ANTIGEN

This assay was performed by an inhouse ELISA in accordance with the method described by Troncone and colleagues, ${ }^{9}$ adding $5 \mathrm{mmol} / 1 \mathrm{CaCl}_{2}$ to $\mathrm{U}$ bottomed microtitre plates according to Sulkanen and colleagues. ${ }^{8}$ Values were expressed as a percentage of positive reference sera, obtained from untreated coeliac patients diagnosed according to the criteria of the European Society of Pediatric Gastroenterology and Nutrition, ${ }^{15}$ showing in all cases the presence of EmA. Anti-tTG values greater than the 95th percentile of the control group, including over 100 healthy controls negative for serum EmA, were considered positive $(8 \%$ of the reference serum). The intra-assay coefficient of variation for the IgA t-TG autoantibody ELISA was $8.7 \%(n=22)$, and the inter-assay coefficient of variation was $10.3 \%(n=18)$.

HUMAN RECOMBINANT TRANSGLUTAMINASE The h-tTG gene was amplified from the intestinal biopsy of a untreated patient with CD using primers specific for the coding region of the gene, as previously described. ${ }^{17}$ Briefly, cDNA was cloned into an expression vector (pET28b; Novagen, Madison, Wisconsin, USA) expressed in bacteria and purified under non-denaturing conditions using IMAC (Qiagen, Valencia, California, USA). Purity of the recombinant protein was assessed by sodium dodecyl sulphate-polyacrylamide gel electrophoresis.

SERUM ANTI-tTG ELISA DETERMINATION USING HUMAN RECOMBINANT tTG AS ANTIGEN

This assay was performed in the laboratory of the paediatric department of the University Hospital of Trieste, on serum samples which had been kept frozen at $-80^{\circ} \mathrm{C}$; a control test 
Table 1 Liver histology findings and viral hepatitis markers in the 98 consecutive hypertransaminasaemia patients included in the study

\begin{tabular}{|c|c|c|c|c|}
\hline & $\begin{array}{l}\text { Anti-HCV+ } \\
\text { HBsAg- }\end{array}$ & $\begin{array}{l}\text { Anti-HCV- } \\
\text { HBsAg+ }\end{array}$ & $\begin{array}{l}\text { Anti-HCV+ } \\
\text { HBsAg+ }\end{array}$ & $\begin{array}{l}\text { Anti-HCV- } \\
\text { HBsAg- }\end{array}$ \\
\hline Normal histology & 1 & - & - & - \\
\hline Steatosis & 3 & 1 & - & 2 \\
\hline Minimal changes & 3 & 2 & - & - \\
\hline Minimal chronic hepatitis & 15 & 5 & - & - \\
\hline Mild chronic hepatitis & 30 & - & 1 & - \\
\hline Moderate chronic hepatitis & 9 & 2 & 1 & - \\
\hline Severe chronic hepatitis & 3 & - & - & - \\
\hline Hepatitis with severe fibrosis & 13 & - & - & - \\
\hline Liver cirrhosis & 5 & - & - & 2 \\
\hline
\end{tabular}

HCV, hepatitis C virus; HBsAg, hepatitis B surface antigen.

performed in that laboratory on 20 serum samples in which anti-tTG antibodies were first assayed on fresh serum and then eight months later after freezing at $-80^{\circ} \mathrm{C}$ showed that the preservation did not significantly alter the results (interassay coefficient of variation was $9.6 \%$ ).

Serum IgA anti-h-tTG antibodies were determined as recently reported, with slight modifications. ${ }^{17}$ The human recombinant antigen was diluted in phosphate buffered saline (PBS) to yield a protein concentration of $10 \mu \mathrm{g} / \mathrm{ml}$. A $0.1 \mathrm{ml}$ aliquot of this solution was placed in each well of a flat bottomed plate (EIA/RIA 2580; Costar, Cambridge, Massachusetts, USA). After an overnight incubation at $4^{\circ} \mathrm{C}$, the plates were washed three times in PBS-0.05\% Tween 20 and blocked with PBS$0.1 \%$ Tween 20 for 20 minutes at room temperature. Serum samples diluted 1:100 in PBS- $0.1 \%$ Tween 20 were incubated for one hour at room temperature. The plates were washed and incubated for one hour at room temperature with 1:4000 phosphatase conjugated antihuman IgA (Sigma A-3062) diluted in PBS- $1 \%$ bovine serum albumin- $4 \%$ polyethylene glycol. The immune reaction was developed by adding substrate solution and adsorbance was read in a microplate reader at $405 \mathrm{~nm}$ until the positive control serum reached an optical density value of 1.9 . Results were expressed as a percentage of the positive control serum. Normal values were taken as $<16 \%$ which represented a value $>2$ SD above the mean of 500 healthy subjects. The intra-assay coefficient of variation for the $\operatorname{IgA}$ h-tTG autoantibody ELISA was $4 \%(n=10)$ and the inter-assay coefficient of variation was $9 \% \quad(\mathrm{n}=10)$. This ELISA method has a sensitivity of $97 \%$ and a specificity of $99 \%$.

\section{INTESTINAL HISTOLOGY}

Biopsy specimens were obtained and orientated as previously described. ${ }^{18}{ }^{19}$ Specimens were embedded in paraffin. Slides were stained with haematoxylin and eosin and graded by conventional histology as normal, partial villous atrophy, and subtotal villous atrophy. Furthermore, a count of the intraepithelial lymphocyte (IEL) population was performed and their number was calculated per number of enterocytes (normal values $<35$ IEL per 100 enterocytes). Histology was described by an examiner unaware of the laboratory test results.

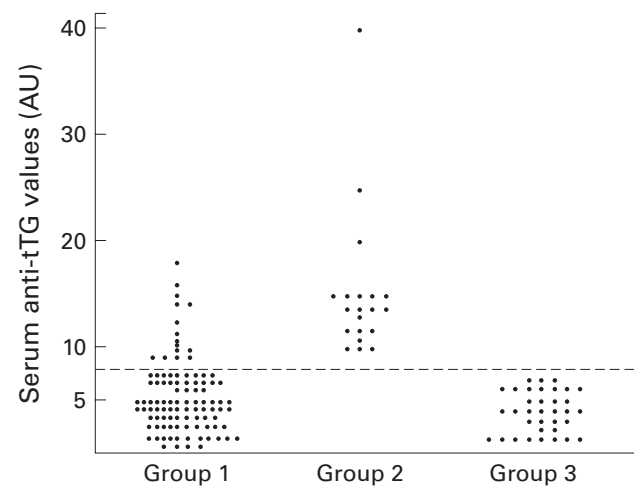

Figure 1 Serum IgA class tissue transglutaminase antibody (anti-tTG) titres determined by ELISA performed using Sigma $t T G$ from guinea pig as the antigen. The results are expressed as $A U$ values. The following groups were studied: group 1 , hypertransaminasaemia patients $(n=98)$; group 2 , coeliac disease patients (EmA positive) on a gluten containing diet $(n=20)$; group 3, healthy control subjects (EmA negative) $(n=35)$. An arbitrary cut off level for positivity (broken line) was drawn at an $A U$ of 8 .

STATISTICAL ANALYSIS

The percentage of anti-tTG and EmA positive results was calculated. Analysis of frequency was performed using the $\chi^{2}$ test. Spearman's $r$ correlation coefficient was used to evaluate the association of serum anti-tTG results with liver histology or laboratory test results. Multiple linear regression analysis was performed to evaluate the association between anti-tTG positivity and serum ALT and AST, serum albumin, serum bilirubin and alkaline phosphatase, serum gamma globulin, serum $\gamma$ glutamyl transferase, and the presence of autoantibodies (ANA, ASMA, AMA, antiLKM1) in chronic liver disease patients.

\section{Results}

Table 1 summarises the liver histology findings and viral hepatitis marker results in all patients studied.

Regarding serological screening for CD, fig 1 shows anti-tTG values obtained with the ELISA based on tTG from guinea pig as the antigen in subjects with chronic liver disease compared with the two control groups; all CD patients with known EmA positivity (CD controls, group 2) had anti-tTG values above the normal limit (range 10-40 AU) whereas all healthy EmA negative controls (group 3) had values within the normal range. In the hypertransaminasaemic patients (group 1), we found 15/98 (16\%) subjects with anti-tTG values above normal; these values were in the same range as those observed in the $\mathrm{CD}$ patients. However, when $\operatorname{IgA} \mathrm{EmA}$ were assayed, only $2 / 98$ ( $2 \%$ ) patients with chronic liver disease were positive; in fact, EmA was negative in all anti-tTG negative hypertransaminasaemic patients but also in 13/15 patients positive for serum IgA anti-tTG.

Nine of 15 hypertransaminasaemic patients positive for anti-tTG, including two EmA positive patients, consented to intestinal biopsy for histological study. Intestinal histology showed subtotal mucosa atrophy in two patients who were positive for both EmA and anti-tTG 
Table 2 Clinical, laboratory, and histological characteristics of the 15 subjects positive for serum IgA anti-tTG antibodies, compared with all other anti-tTG negative hypertransaminasaemia patients

\begin{tabular}{|c|c|c|c|c|c|c|c|c|c|}
\hline Case No & $\begin{array}{l}\text { Anti-tTG } \\
(A U)\end{array}$ & $E m A$ & $\begin{array}{l}\text { Natural } \\
\text { autoantibodies }\end{array}$ & $\begin{array}{l}A L T / A S T \\
(U I / L)\end{array}$ & Liver histology & $\begin{array}{l}\text { Intest histology } \\
\text { (villi/crypts) }\end{array}$ & $\begin{array}{l}\text { Intestinal } \\
\text { histology (IEL) }\end{array}$ & $\begin{array}{l}\text { Associated } \\
\text { diseases }\end{array}$ & Viral markers \\
\hline 1 & 17 & + & ANA+ & $40 / 46$ & MILH & 0.8 & 63 & - & HCV+ \\
\hline 2 & 14 & + & ASMA+ & $60 / 84$ & $S$ & 1.1 & 71 & IDDM & $\mathrm{HCV}-/ \mathrm{HBV}-$ \\
\hline 3 & 14 & - & ASMA+ & $39 / 98$ & $\mathrm{MH}$ & 3.3 & 14 & - & $\mathrm{HCV}+$ \\
\hline 4 & 10.5 & - & ANA+ & $177 / 221$ & SF & 3.4 & 19 & - & $\mathrm{HCV}+$ \\
\hline 5 & 8.7 & - & LKM1+ & $67 / 75$ & MODH & 4.0 & 30 & - & HBV+ \\
\hline 6 & 11 & - & None & $924 / 698$ & SF & 2.9 & 27 & - & $\mathrm{HCV}+$ \\
\hline 7 & 9.4 & - & None & $84 / 80$ & MH & 3.6 & 31 & - & $\mathrm{HCV}+$ \\
\hline 8 & 13 & - & ANA+ & $165 / 117$ & LC & 3.5 & 28 & CG & $\mathrm{HCV}-/ \mathrm{HBV}-$ \\
\hline 9 & 9 & - & None & $47 / 49$ & $\mathrm{MH}$ & Not perf. & Not perf. & - & $\mathrm{HCV}+$ \\
\hline 10 & 15 & - & $\mathrm{AMA}+\mathrm{LKM} 1+$ & $31 / 53$ & $\mathrm{MH}$ & Not perf. & Not perf. & - & HBV+ \\
\hline 11 & 8.4 & - & ANA+ & $116 / 162$ & $\mathrm{MH}$ & Not perf & Not perf. & - & $\mathrm{HCV}+$ \\
\hline 12 & 9.7 & - & ASMA+ ANA+ & $121 / 170$ & SF & Not perf & Not perf. & Thyroiditis & $\mathrm{HCV}+$ \\
\hline 13 & 8.8 & - & ANA+ AMA+ & $89 / 95$ & MH & Not perf & Not perf. & - & $\mathrm{HCV}+$ \\
\hline 14 & 16 & - & None & $42 / 44$ & $\mathrm{~S}$ & 2.9 & 17 & - & HCV-/HBV- \\
\hline 15 & 9.9 & - & ANA+ & $84 / 106$ & $\mathrm{~S}$ & Not perf & Not perf. & - & $\mathrm{HBV}+$ \\
\hline \multirow{10}{*}{$\begin{array}{l}\text { Anti-tTG negative } \\
\text { controls }\end{array}$} & Range $2-7.2$ & $83 / 83$ neg & ASMA +2 cases, & & $\mathrm{N}=1$ & Not perf. & Not perf. & $\mathrm{CG}=4$, & $\mathrm{HCV}+=73$ \\
\hline & & & $\mathrm{AMA}+2$ cases, & & $S=3$ & & & IDDM=2, & $\mathrm{HBV}+=7$ \\
\hline & & & $\mathrm{ANA}+4$ cases, & & $\mathrm{MCHS}=5$ & & & NIDDM=3, & $\mathrm{HCV}+1$ \\
\hline & & & LKM $1+2$ cases & & $\mathrm{MH}=14$ & & & $\mathrm{PU}=6$, & $\begin{array}{l}\mathrm{HBV}+=2 \\
\mathrm{HCV}-1\end{array}$ \\
\hline & & & LeVtrit 2 cases & & & & & & $\mathrm{HBV}-=1$ \\
\hline & & & & & MILH=30 & & & $\mathrm{CVD}=3$ & \\
\hline & & & & & $\mathrm{MODH}=11$ & & & & \\
\hline & & & & & $\mathrm{SH}=3$ & & & & \\
\hline & & & & & $\mathrm{SF}=10$ & & & & \\
\hline & & & & & $\mathrm{LC}=6$ & & & & \\
\hline
\end{tabular}

EmA, antiendomysial antibody; tTG, tissue transglutaminase; AST, aspartate aminotransferase; ALT, alanine aminotransferase; HCV, hepatitis C virus; HBV, hepatitis B virus; ANA, antinuclear antibodies; AMA, antimitochondrial antibodies; ASMA, antismooth muscle antibodies; LKM, anti-liver-kidney microsomal antibodies; IEL, intraepithelial lymphocyte.

Liver histology: N, normal; S, steatosis; MCHS, minimal changes; MH, minimal chronic hepatitis; MILH, mild chronic hepatitis; MODH, moderate chronic hepatitis; $\mathrm{SH}$, severe chronic hepatitis; SF, hepatitis with severe fibrosis; LC, liver cirrhosis.

Associated diseases: CG, congestive gastropathy; IDDM, insulin dependent diabetes mellitus; NIDDM, non-insulin dependent diabetes mellitus; PU, peptic ulcer; CVD, cardiovascular disease.

Normal reference values: anti-t-TG <8 AU; EmA: serum dilution <1:5; natural autoantibodies: serum dilution <1:80; ALT and AST <40 UI/1; villi/crypts ratio >2.8; IEL count $<35 / 100$ enterocytes.

(villi/crypts ratio $0.8-1.1$ ) with a marked increase in IELs. However, none of the other seven subjects positive for serum IgA anti-tTG (but EmA negative) had intestinal mucosa abnormalities: all had a normal villi/crypts ratio (range 2.9-4) and in all the IEL count was below the normal limit for our laboratory.

Table 2 shows the clinical data, liver and intestinal histology, and laboratory characteristics of the two EmA+/anti-tTG+ and the other $13 \mathrm{EmA}-/$ anti-tTG+ patients. The $13 \mathrm{EmA}-/$ anti-tTG+ patients did not differ from the other 83 anti-tTG- hypertransaminasaemic subjects for the presence of gastrointestinal symptoms or associated diseases: none had overt malabsorption syndrome and no difference in body mass index was observed. Severity of liver disease evaluated from liver histology was identical in both groups, and the distribution of viral hepatitis markers was also similar. However, there was a significantly higher frequency of patients with positive serum autoantibodies (ANA, AMA, ASMA, antiLKM) in the anti-tTG+ group than in the other subjects $\left(9 / 13 \quad v 10 / 84 ; \quad \chi^{2}=9.82\right.$, $\mathrm{p}<0.003)$ and serum ANA titres correlated with anti-tTG values (Spearman's $r$ correlation coefficient $0.78 ; \mathrm{p}<0.0001$ ). There was no correlation between serum anti-tTG values and most of the other laboratory parameters evaluated (serum albumin, serum ALT and AST, serum bilirubin and alkaline phosphatase, prothrombin activity, serum $\gamma$ glutamyl transferase, and platelet count) but a statistically significant positive correlation was found between serum gamma globulin and anti-tTG values (Spearman's $r$ correlation coefficient 0.33 ; $\mathrm{p}<0.01)$. Finally, we performed a multiple linear regression analysis which describes the relationship between a dependent variable, such as positivity of serum anti-tTG in the ELISA based on tTG from guinea pig as the antigen, and various independent laboratory variables; this analysis confirmed the significant association between serum anti-tTG positivity and two of the independent variables examined-serum autoantibody positivity (beta value $0.566, p<0.0004$ ) and serum gamma globulin (beta value 0.465, p<0.008). There was no correlation between anti-tTG positivity and the other independent variables examined $\left(r^{2}=0.46\right)$.

Eight months after the conclusion of the first part of the study, we performed an ELISA for IgA anti-tTG antibodies using h-tTG as the antigen, and the sera of all patients included in this study were re-evaluated. In the patient group with chronic liver disease, only the two subjects with atrophy of the intestinal mucosa and serum EmA+ at the moment of diagnosis had elevated anti-tTG antibodies: their values were in the range of those observed in patients with CD (19\% and $32.1 \%$, respectively). All other patients with chronic liver disease had anti-tTG values within the normal limit (range 1-13.1\%), including the 13 subjects with false positive anti-tTG values when evaluated with the ELISA based on tTG from guinea pig as the antigen. Finally, in the serum of these 13 patients we repeated the anti-tTG ELISA based on tTG from guinea pig as the antigen: 
in all cases a false positive result was confirmed. For the control groups, all CD patients with known $\mathrm{EmA}+$ were positive for serum IgA anti-tTG (range 17-116\%) whereas none of the healthy controls were positive (range $1-12.3 \%$ )

The two patients with subtotal intestinal mucosa atrophy began a gluten free diet and after 4-5 months of this diet showed negative serum IgA EmA and anti-tTG; in one of these patients, who was anti-HCV positive, hypertransaminasaemia persisted on a gluten free diet. In the other patient, negative for viral hepatitis markers, hypertransaminasaemia disappeared after three months on a gluten free diet. None of the patients positive for anti-tTG but EmA- commenced a gluten free diet.

\section{Discussion}

It is known that CD can cause chronic hypertransaminasaemia both in adults ${ }^{35}$ and in children $^{2420}$ and the relative risk for CD in patients with chronic unexplained hypertransaminasaemia compared with the general population has been estimated as $18.6 .^{21} \mathrm{~A}$ previous study indicated that in patients with chronic liver diseases, serum EmA determination is useful for CD diagnosis. ${ }^{6}$ However, the EmA assay has several limitations: interpretation of the immunofluorescence pattern is subjective, it requires monkey oesophagus or human tissues as substrate, and it is not easy to use in large scale screening. Thus the recent identification of the protein tTG as the autoantigen of $\mathrm{CD}^{7}$ has made possible large scale use of an ELISA test, based on tTG antigen from guinea pig liver, to detect IgA class anti-tTG antibodies.

Previous studies on IgA anti-tTG determination have indicated a very high specificity of this ELISA assay for CD diagnosis, and it has been suggested that the false positive results could be latent CD cases $^{7-9}$; hence the use of the ELISA based on this protein has been promoted to screen large populations. ${ }^{11}$ However, none of the previous studies included patients with chronic liver disease of different causes and, before the present study, we had no data on the use of the anti-TG antibody assay in these patients. Our data clearly indicate that serum IgA anti-TG ELISA determination based on tTG from guinea pig liver as the antigen is not useful in screening patients with chronic liver disease for CD. In fact, we found that all patients with positive anti-tTG antibodies and negative EmA, who agreed to undergo intestinal biopsy ( $7 / 13$ subjects), had normal intestinal histology, with normal villi and crypts and no increase in IELs, which is considered a marker of latent CD. ${ }^{22}$ Thus it is evident that the current ELISA based on guinea pig tTG is unspecific when used for $\mathrm{CD}$ diagnosis in patients with chronic liver disease.

Regarding the cause of these "false positive" anti-tTG tests, we demonstrated that this phenomenon was clearly linked to the purity of the antigen used in the currently advised anti-tTG ELISA. In fact, this widely used ELISA is based on tTG which is a crude extract from guinea pig liver; it has been verified that Sigma tTG is composed of at least 14 different components ${ }^{23}$ and it is logical to hypothesise that in the sera of patients with chronic liver disease there could be antibodies for protein antigens from the liver matrix, probably common (or cross reacting) to humans and other mammals. In fact, in the second part of the study when we were able to test our sera for anti-tTG using a pure human recombinant tTG, we did not observe any false positive results: all 13 subjects with false positive anti-tTG values when evaluated with the ELISA based on tTG from guinea pig as antigen resulted in negative values with the new ELISA system. This confirms that human tTG ELISA has a higher diagnostic accuracy in CD diagnosis than the commonly used anti-tTG ELISA based on guinea pig antigen. However, it must be remembered that all gastroenterogical studies published to date $\mathrm{e}^{7-11}$ which reported a very high specificity of anti-tTG determination for CD diagnosis were performed with the same ELISA used in the first part of the present study (based on tTG from guinea pig liver) and that this method has been suggested for large scale CD screening. ${ }^{81011}$ Our findings appear to be linked to the presence of chronic liver disease and/or hyperglobulinaemia and we emphasise that such a high frequency of false positive anti-tTG results cannot be extrapolated to the general population.

In conclusion, we found a high frequency of false positive anti-tTG test results in patients with chronic liver disease using the commonly used anti-tTG ELISA based on tTG from guinea pig liver as antigen. The presence of anti-tTG did not correlate with the presence of EmA or CD. We demonstrated that these false positive results were due to the "nature" of the antigen used in the ELISA system and that they disappeared if a new ELISA, based on human recombinant tTG as antigen, was employed. Thus the anti-human-tTG ELISA, and not the anti-tTG assay based on tTG from guinea pig as the antigen, must be used as a screening tool for CD in patients with chronic liver disease.

This work was supported by a grant to G Montalto, $60 \%$ from MURST, and by grant 12/99 from IRCCS "Burlo Garofalo".

1 Hagander B, Brandt L, Sjolund K, et al. Hepatic injury in celiac disease. Lancet 1977;2:270-2.

2 Bonamico M, Pitzalis G, Culasso F. Hepatic damage during coeliac disease in childhood. Minerva Pediatr 1986;38:95962.

3 Bardella MT, Fraquelli M, Quatrini M, et al. Prevalence of hypertransaminasemia in adult celiac patients and effect of gypertransaminasemia in adult celiac patien

4 Carroccio A, Iannitto E, Cavataio F, et al. Sideropenic anemia and celiac disease: one study, two points of view. Dig Dis Sci 1998;43:673-8.

5 Gonzalez-Abraldes J, Sanchez-Fueyo A, Bessa X, et al. Peristent hypertransaminasemia as the presenting feature of celiac disease. Am F Gastroenterol 1999;94:1095-7.

6 Sjoberg K, Lindgren S, Eriksson S. Frequent occurrence of non-specific gliadin antibodies in chronic liver disease. Endomysial but not gliadin antibodies predict coeliac disease in patients with chronic liver disease. Scand $\mathcal{F}$ Gastroenterol 1997;32:1162-7.

7 Dieterich W, Ehnis T, Bauer M, et al. Identification of tissue transglutaminase as the autoantigen of celiac disease. Nat Med 1997;3:797-801.

8 Sulkanen S, Halttunen $\mathrm{T}$, Laurila $\mathrm{K}$, et al. Tissue transglutaminase autoantibody enzyme-linked immunosorbent assay in detecting coeliac disease. Gastroenterology $1998 ; 115: 1322-8$. 
9 Troncone R, Maurano F, Rossi M, et al. IgA antibodies to tissue transglutaminase: an effective diagnostic test for tissue transglutaminase: an effective diagn

10 Brusco G, Izzi L, Corazza GR. Tissue transglutaminase antibodies for coeliac disease screening. Ital $\mathcal{F}$ Gastroenterol Hepatol 1998:30:496-7.

11 Dieterich W, Laag E, Schopper H, et al. Autoantibodies to tissue transglutaminase as predictors of celiac disease. Gastroenterology 1998;115:1317-21.

12 Habior AB, Lewartowska A, Orlowska J, et al. Autoantibodies to tissue transglutaminase are not a marker of celiac disease associated with primary biliary cirrhosis. Hepatology 1999;30:474A

13 Soresi M, Carroccio A, Bonfissuto G, et al. Ultrasound detection of abdominal lymphadenomegaly in subjects with hepatitis $\mathrm{C}$ virus infec transaminases. A predictive index of liver histology severity. 7 Hepatol 1998;28:544-9.

14 Desmet VJ, Gerber M, Hoofnagle JH, et al. Classification of chronic hepatitis: diagnosis, grading, staging. Hepatology 1994; 19:1513-20.

15 Walker-Smith JA, Guandalini S, Schmitz J, et al. Revised criteria for diagnosis of coeliac disease. Arch Dis Child 1990;65:909-11.
16 Carroccio A, Cavataio F, Iacono G, et al. IgA antiendomysial Carroccio A, Cavataio $\mathrm{F}$, Iacono $\mathrm{G}$, et al. IgA antiendomysial antibodies on the umbilical cord in diagn

17 Sblattero D, Berti I, Trevisiol C, et al. Human recombinant tissue transglutaminase ELISA: an innovative diagnostic assay for coeliac disease. Am f Gastroenterol 2000;95:12537.

18 Carroccio A, Iacono G, Lerro P, et al. Role of pancreatic impairment in growth recovery during gluten-free diet in childint in rentroentogy 1997:112:1839-4

19 Perera DR, Weinstein WM, Rubin CE Small intestinal biopsy. Hum Pathol 1975;6:157-217.

20 Vajro P, Fontanella A, Mayer M, et al. Elevated serum aminotransferase activity as an early manifestation of gluten-sensitive enteropathy. F Pediatr 1993;122:416-19.

21 Bardella MT, Vecchi M, Conte D, et al. Chronic unexplained hypertransaminasemia may be caused by occult celiac disease. Hepatology 1999;29:654-7.

22 Marsh MN. The mucosal pathology in gluten sensitivity. In: Marsh MN, ed. Coeliac disease. Oxford: Blackwell Scientific, 1992:136-91.

23 Maki M. Tissue transglutaminase as the autoantigen of coeliac disease. Gut 1997;41:565-6.

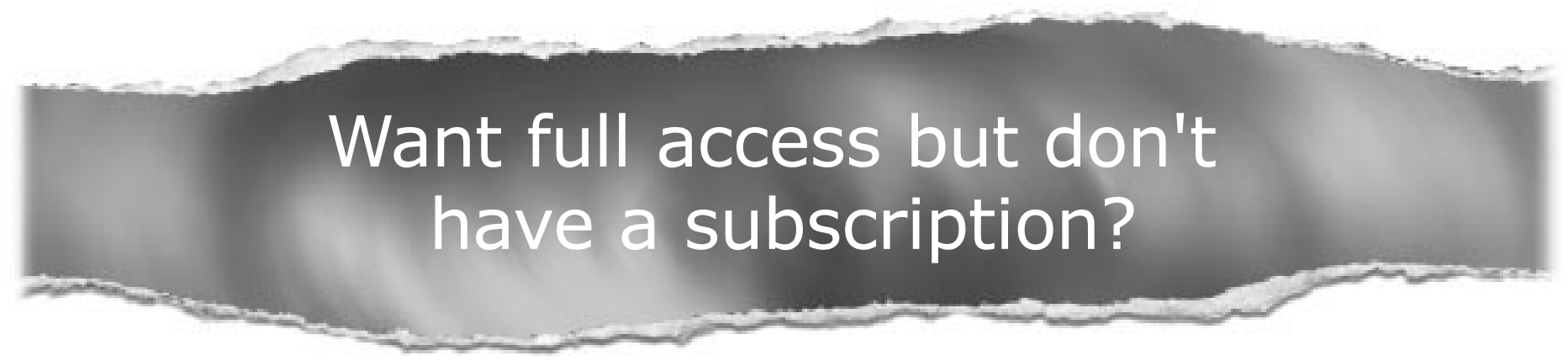

\section{Pay per access}

For just US $\$ 25$ you can have instant access to the whole website for 30 days. During this time you will be able to access the full text for all issues (including supplements) available. You will also be able to download and print any relevant pdf files for personal use, and take advantage of all the special features Gut online has to offer.

www.gutjnl.com 